\title{
Scanning for a Satellite Radar Wind Sounder (RAWS)
}

\author{
R.K Moore, B. Beh, and S. Song \\ University of Kansas, Radar Systems and Remote Sensing Laboratory \\ 2291 Irving Hill Road, Lawrence, KS 66046-2969 USA \\ TEL: 913/864-4835 , FAX: 913/864-7789 , E-MAIL: graham@ardneh.rsl.ukans.edu
}

\begin{abstract}
The Radar Wind Sounder (RAWS) is a proposed satellite-borne radar capable of measuring wind aloft using Doppler shifts of hydrometeors. Previous studies showed the sensor can retrieve wind vectors at various levels in the atmosphere while also measuring rain rates and ocean-surface winds. Here we present the method of retrieving wind-aloft information from a conically scanned antenna. The approach combines the radial velocities measured at many points within a region into a least-squares algorithm to obtain a mean wind for the region.
\end{abstract}

\section{INTRODUCTION}

Analysis and forecasting using numerical weather models would improve greatly if world wide wind information were available. Lasers on satellites were proposed for this purpose, and the Laser Wind Sounder (LAWS) was an original component of the Earth Observation System (EOS) [1]. However, gaps would exist in coverage as lasers can only profile winds in clear air or light clouds. Accordingly, we proposed a radar sensor to complement the laser sensor by profiling winds in the cloudy and rain areas [2,3]. Both systems measure Doppler shift, from aerosols for lasers and from hydrometers for radars.

Our previous studies showed that the optimal frequency for single-channel coverage is $35 \mathrm{GHz}$ and, $24 \mathrm{GHz}$ and $94 \mathrm{GHz}$ for dual channels [4]. Using these frequencies, RAWS can cover most cloud types with the original proposed power ( 3 $\mathrm{kW}$ peak), and miss the thin clouds with reduced power [5]. In clear air or light clouds, the contributions from the vertical components of wind vectors are small and can therefore be ignored in the calculation of the wind vectors. In precipitating systems, the vertical components can be estimated from the raindrop fall speed, based on the rain-echo intensity [6].

This paper considers the derivation of the horizontal wind vectors and the accuracy achievable by integrating multiple measurements of radial velocities. The approach considered is to measure radial velocities at many points within a region, combining the results in a least-squares algorithm to obtain a mean wind for the region.

\section{SCAN PATTERN AND MEASURED POINTS DISTRIBUTION}

Fig. 1 shows the measured points distribution by a conically scan antenna using 256 pulses per point. The figure only displays half of the swath. The asterisks are the forward looks and the circles are the aft looks. Note that the measurements closer to the edge area are much more dense than that in the center area. These measured points are grouped to form cells of $66 \mathrm{~km}$ square. The exact dimensions of the cells were chosen based on the assumed scan rate to allow equal numbers of measurements in each cell within a region.

\section{DERIVATION OF WIND VECTORS FROM THE MEASURED DOPPLER VELOCITY}

The wind vector can be expressed by

$$
\overline{U_{w}}=U_{w x} \bar{X}+U_{x y} \bar{Y}+U_{w z} \bar{Z}
$$

where $U_{w z}$ can be derived from the rain fall speed [6] and will be ignored in the simulation. The radial velocities from the measured points within a cell are given by

$$
\begin{array}{r}
U_{r i}=U_{w x} \sin \theta \cos \phi_{i}+U_{w y} \sin \theta \sin \phi_{i} \\
i=1,2, \ldots, N
\end{array}
$$

Applying a least-squares method to (2) we obtained [7]

$$
\left(\begin{array}{l}
U_{w x} \\
U_{w y}
\end{array}\right)=\Delta\left(\begin{array}{cc}
\sum \sin ^{2} \phi_{i} & -\sum \sin \phi_{i} \cos \phi_{i} \\
-\sum \sin \phi_{i} \cos \phi_{i} & \sum \cos ^{2} \phi_{i}
\end{array}\right)\left(\begin{array}{l}
\sum U_{d i} \cos \phi_{i} \\
\sum U_{d i} \sin \phi_{i}
\end{array}\right)
$$

where

$$
\Delta=\frac{1}{\sin \theta \sum \sin ^{2} \phi_{i}-\left(\sum \sin \phi_{i} \cos \phi_{i}\right)^{2}}
$$

The standard deviation of the average velocity for a covariance estimator is [8]

$$
\begin{aligned}
\Delta V_{d}= & \lambda\left[[ 3 2 \pi ^ { 2 } M \rho ^ { 2 } ( T _ { s } ) T _ { s } ^ { 2 } ] ^ { - 1 } \left[\left[\left(1-\rho^{2}\left(T_{s}\right)\right) \lambda\right]\right.\right. \\
& \left.\left.\frac{1}{\left[4 \sigma_{s} T \Pi^{1 / 2}\right]}+N^{2} / S^{2}+2(N / S)\right]\right]^{1 / 2}
\end{aligned}
$$

where $M$ is the number of pulses per measured point, $T_{s}$ is the interval between pulses in a pulse-pair, $T$ is the pulse-pair repetition time, $\sigma_{s}^{2}$ is the variance of the power spectral densities of received echoes, and N/S is the inverse of signalto-noise ratio. $\rho\left(T_{s}\right)$ is the correlation function [5]

$$
\mathrm{e}\left(m T_{s}\right)=\exp \left[-8\left(\frac{\pi \sigma_{s} m T_{s}}{\lambda}\right)^{2}\right]
$$

where $m=1$ and $\lambda$ is the RF wavelength. $\sigma_{s}$ is bounded by [5] 


$$
\sigma_{x} \leq \frac{\lambda}{4 \pi T_{s}}
$$

to prevent $\Delta V_{d}$ from increasing exponentially and also to keep the samples correlated.

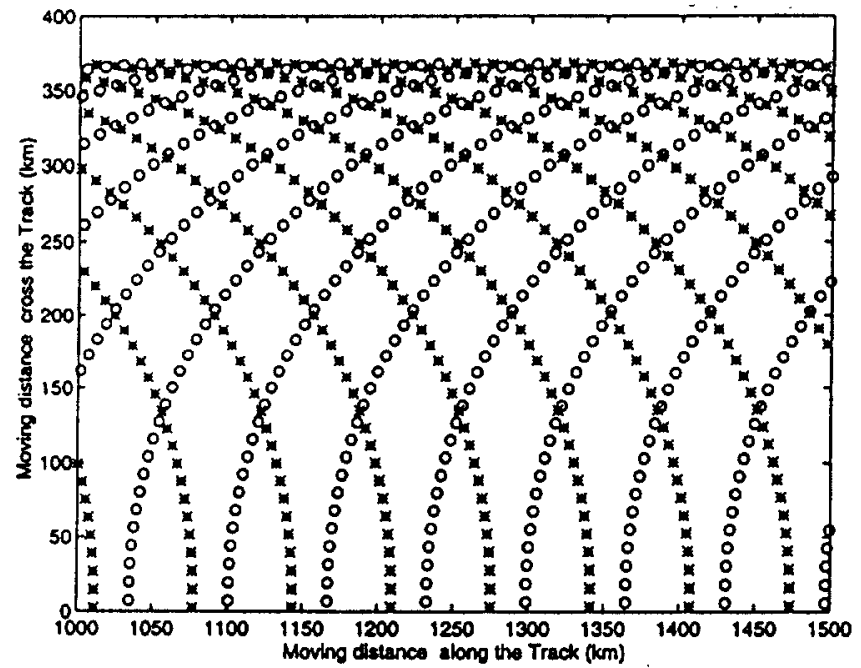

Fig. I Conically scanned measured point distributions on the ground.

Using (3) and (5), along with the measurement errors (assuming Gaussian), we developed an algorithm to simulate the errors in the measured wind vectors. The simulations assumed 256 pulses per point and an SNR of $10 \mathrm{~dB}$. The cell sizes are $66 \mathrm{~km} \mathrm{x} 66 \mathrm{~km}$ in areas close to the edge (bands \# $1,2,7,8)$ and $132 \mathrm{~km} \times 132 \mathrm{~km}$ at areas around the center (bands $\# 3,4,5,6$ ). Our preliminary simulations showed that we need a larger cell for the center areas to keep the errors below the specifications [7].

Figs. 2 to 6 depict the rms errors obtained from the simulations. Here we only present the results for wind speed at 5 $\mathrm{m} /$ and $14 \mathrm{~m} / \mathrm{s}$, as we found that both the magnitude and direction errors tend to decrease as the wind speeds increase [7]. At $5 \mathrm{~m} / \mathrm{s}$, the direction errors at the center bands are slightly above $20^{\circ}$ for $24-\mathrm{GHz}$ cases and below $20^{\circ}$ for the $35-\mathrm{GHz}$ cases. The magnitude errors are less than $2 \mathrm{~m} / \mathrm{s}$ for all the cases except the $24-\mathrm{GHz}$ with wind direction at $90^{\circ}$. At $14 \mathrm{~m} / \mathrm{s}$, both the direction and magnitude errors are below $20^{\circ}$ and $2 \mathrm{~m} / \mathrm{s}$ in all cases except the $24-\mathrm{GHz}$ case with wind direction at $90^{\circ}$, where the magnitude error is slightly above $2 \mathrm{~m} / \mathrm{s}$ at the center bands.

\section{CONCLUSIONS AND RECOMMENDATIONS}

The simulation results indicate that this approach to analyzing RAWS data can provide measurements of wind aloft with adequate accuracy for use in global atmospheric models. However, our simulations assumed the cells to be covered entirely by hydrometeors, which is seldom the case in reality, as the areas involved are relatively large. Using RAWS alone may degrade the measurement accuracy when the cells are only partially covered with cloud. Hence, we recommend the combination of measurements from both the lidar and the radar. The lidars proposed use a conical scan pattern similar to that of the radar, but with a different scan rate. The measurement errors would then differ from those of the radar, but we would expect the results to be similar. When vertical components are significant in rain, we can estimate the vertical speed from the relation between rain rate and fall speed [6]. Thus the combined radar and laser sensors would have the capability to measure winds at most altitudes even in rainy areas.

\section{REFERENCES}

[1] R. J. Curran, Ed., Earth Observation System Instrument Panel Report: LAWSLaserAtmosphericWindSounder, vol.IIg,NASA, Wash.DC, 1987.

[2] W. Xin, A Satellite-Based Radar Wind Sounder, Ph.D. Dissertation, Univ. of Kansas, 1990.

[3] R. K. Moore, M. Stuart, W. Xin, and T. Propp, "A satellite-borne radar wind sensor (RAWS)," Digest IGARSS'92, Houston, pp. 999-1001, 1992.

[4] M. A. Stuart and R. K. Moore, "Satellite-based radar wind sounder (RAWS) optimal frequency study," Digest IGARSS'93, Tokyo, Japan, pp. $215-217,1993$.

[5] M. A. Stuart, Radar Sensitivity and Antenna Scan Pattern Study for a Satellite-Based Radar Wind Sounder (RAWS), M.S. Thesis, Univ. of Kansas, 1992.

[6] S. R. Pallikala and R. K. Moore, "Use of radar wind sensor to determine vertical drop speed for RAWS," RSL Technical Report 10240-1, Radar Systems \& Remote Sensing Laboratory, Univ, of Kansas, May 1994.

[7] S. Song, B. Beh, and R. K. Moore, "Scan patterns and accuracy of a radar wind sensor (RAWS)," RSL Technical Report 11170-2, Radar Systems \& Remote Sensing Laboratory, Univ. of Kansas, Nov. 1995.

[8] R. J. Doviak and D. S. Zmic, Doppler Radar and Weather Observations, Academic Press, New York, 1984.
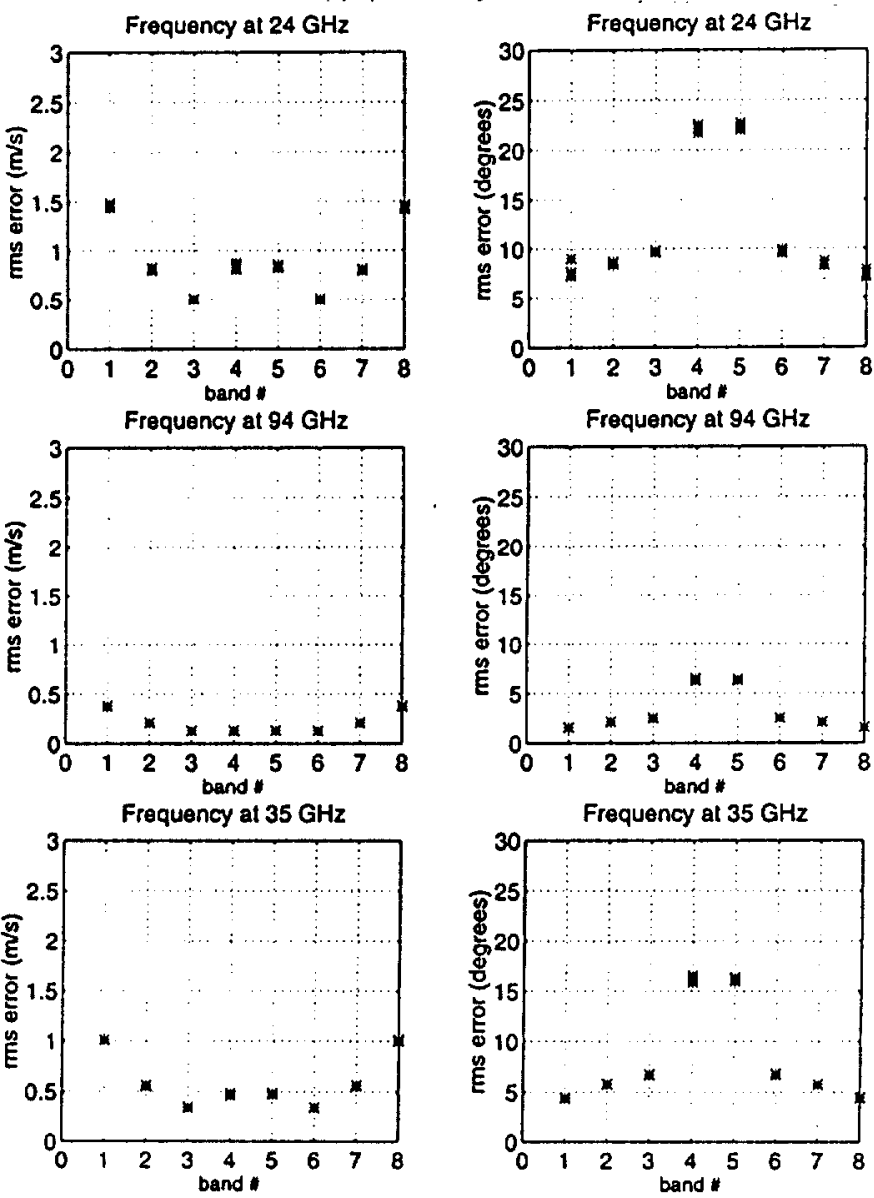

Fig. 2 RMS errors of wind vectors assuming true wind of $5 \mathrm{~m} / \mathrm{s}$ at $0^{\circ}$. 

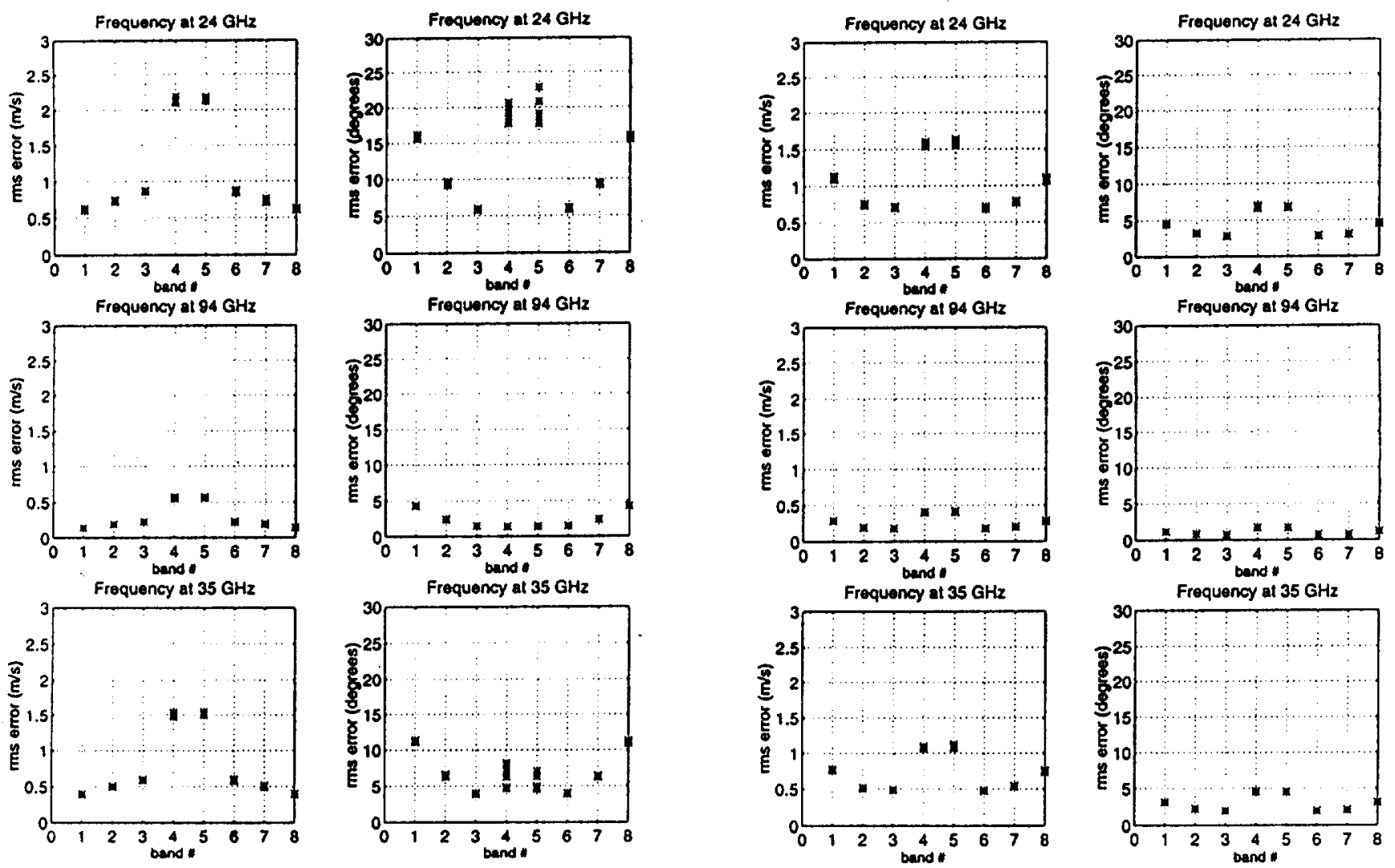

Fig. 3 RMS errors of wind vectors assuming true wind at 5 $\mathrm{m} / \mathrm{s}$ at $90^{\circ}$.

Fig. 5 RMS errors of wind vectors assuming true wind of $14 \mathrm{~m} / \mathrm{s}$ at $45^{\circ}$.
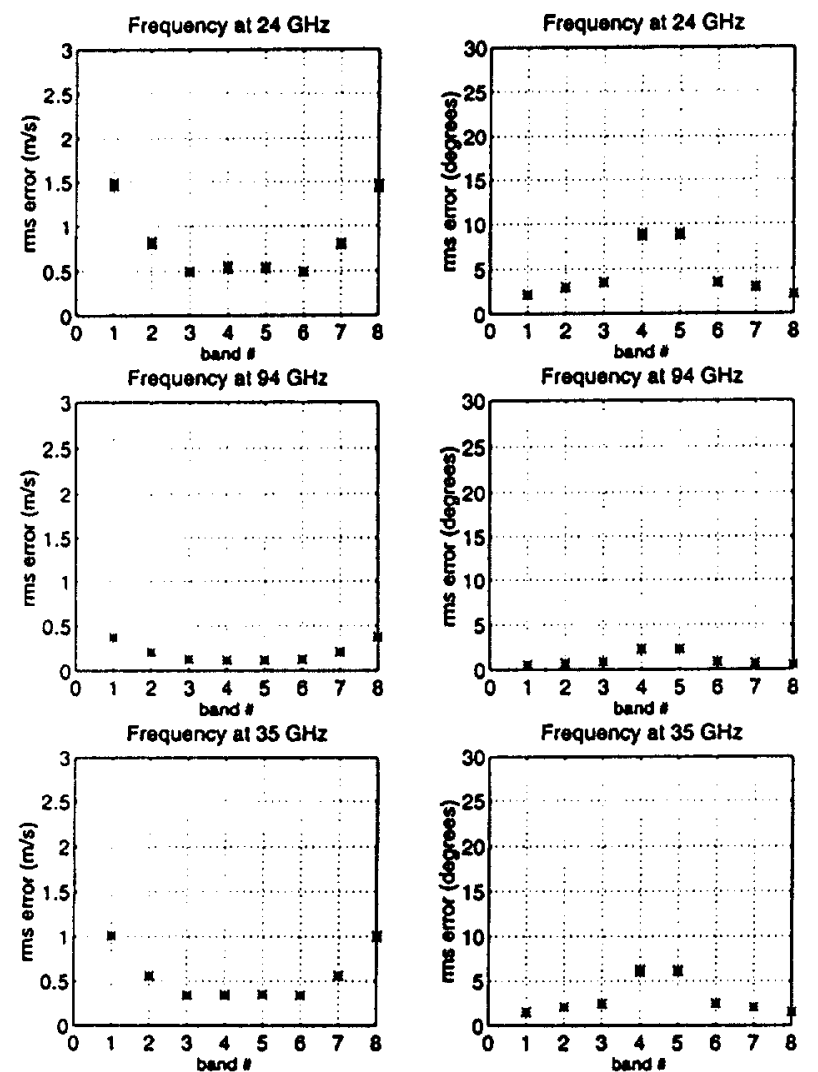

Fig. 4 RMS errors of wind vectors assuming true wind of $14 \mathrm{~m}$ 's at $0^{\circ}$.
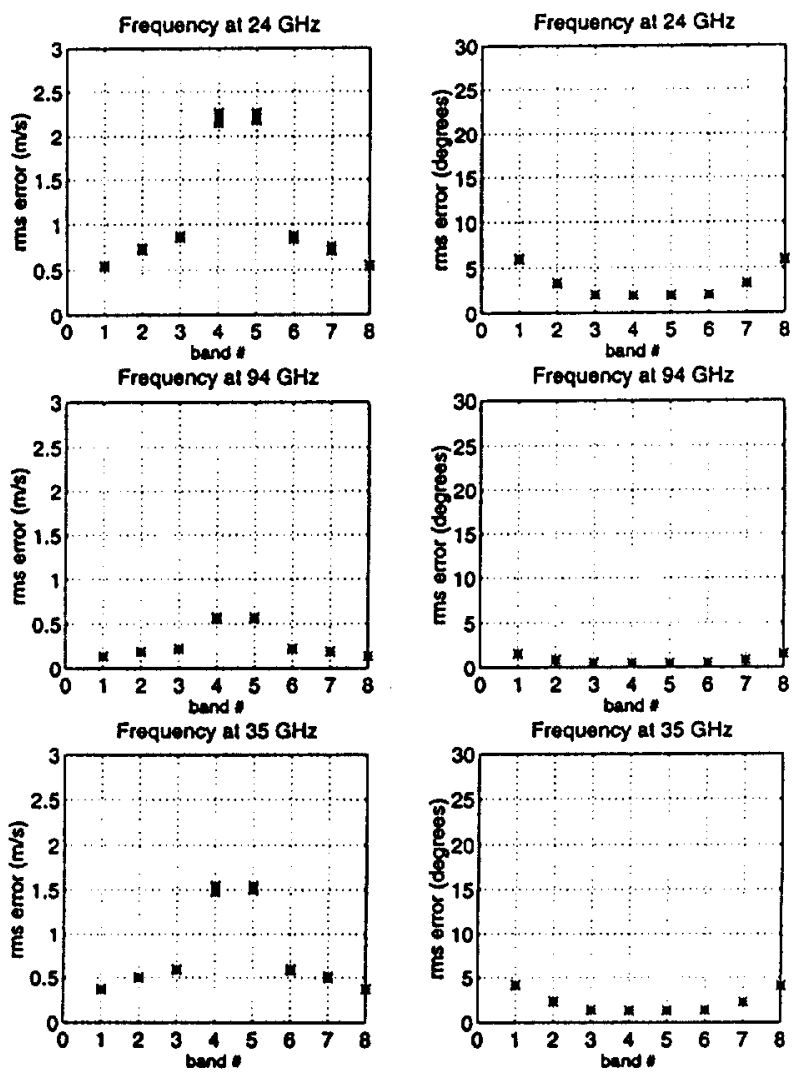

Fig. 6 RMS errors of wind vectors assuming true wind of $14 \mathrm{~m} / \mathrm{s}$ at $90^{\circ}$. 